\title{
Spectrum Sensing using Single-Radio Switched-Beam Antenna Systems
}

\author{
Elpiniki P. Tsakalaki $\ddagger^{\ddagger}$, David Wilcox*, Elisabeth de Carvalho ${ }^{\ddagger}$, Constantinos B. Papadias ${ }^{\dagger}$ and \\ Tharmalingam Ratnarajah* \\ Email: et@es.aau.dk \\ ${ }^{\dagger}$ Broadband, Wireless \& Sensor Networks Group, Athens Information Technology (AIT) \\ ${ }^{\ddagger}$ Department of Electronic Systems, Aalborg University (AAU) \\ ${ }^{*}$ The Institute of Electronics, Communications and Information Technology, Queen's University of Belfast
}

\begin{abstract}
The paper describes spectrum sensing using singleradio switched-beam arrays with reactance-loaded parasitic elements. At a given frequency, the antenna's loading conditions (reactive loads) are optimized for maximum average beamforming gain in the beampattern look direction. Circular permutations of the reactive loads rotate the narrowband beampattern to different angular positions dividing the whole space around the cognitive receiver into several angular subspaces. The beampattern directionality leverages the performance of spectrum sensing algorithms like the energy detection by enhancing the receive signal-to-noise ratio. Moreover, the selection combining across the weakly correlated beampatterns gives rise to a diversity action further boosting the probability of detection.
\end{abstract}

Index Terms-Cognitive radio, spectrum sensing, smartantennas, parasitic arrays, switched-beam antennas, energy detection.

\section{INTRODUCTION}

The cognitive radio (CR) has been introduced as a novel technology paradigm toward efficient, dynamic and flexible usage of the scarce radio spectrum resource [1]. Spectrum sensing is an essential component of a CR system aiming to obtain awareness regarding the spectrum occupancy and the primary user (PU) activity in a specific region. The key role of spectrum sensing is to identify transmission opportunities for the secondary users (SUs) by detecting unoccupied spectrum segments with as much accuracy and low latency as possible.

Several approaches and enabling algorithms of spectrum sensing for CR applications have been proposed, each having different operational requirements, advantages and disadvantages [2]. For example, matched filtering detection [3] needs to know the waveforms and the channels of the PUs whereas cyclostationary detection [4] requires knowledge of the cyclic frequencies of the PUs being sensitive to timing and frequency mismatch. Blind algorithms such as energy detection-based methods [5]- [7] are susceptible to noise power uncertainty but require no a priori knowledge of the PU signals and are quite popular due to their low complexity and simplicity. Techniques

This work has been performed in the context of collaboration between AAU/Center for Teleinfrastruktur and AIT for the joint offering of a Doctoral Program. The work was supported by the Future and Emerging Technologies (FET) Programme within the Seventh Framework Programme for Research of the European Commission, under FET-Open grant CROWN-233843. like cooperative eigenvalue-based sensing [8] or multiantennabased detection [9] are immune to noise uncertainty but increase the sensing complexity.

The use of smart antennas and beamforming systems in CR is another promising direction toward increasing the spectral reuse and improving the sensing performance. A directional beampattern is able to focus the energy in specific angles limiting the interference to unwanted directions and enabling a denser network deployment. In this context, [10] studies the maximum number of users that can be allocated in the same frequency in a CR network using transmit and receive beamforming. In [11], the SU transmitter is equipped with a smart antenna having multiple active elements so as to construct a transmission beampattern that is non-intrusive to the PU link. Further, [12] studies the improvement to the cyclostationary-based detector performance exploiting smartantenna radio receivers using certain beamforming algorithms.

Although most of the existing smart-antenna CR techniques rely on systems with multiple active antenna elements where each element is connected to a separate radio-frequency (RF) chain, the authors in [13], [14] propose spatial-spectrum sensing for scanning both frequency and spatial (angular) resource dimensions via a single-RF frontend. The CR antenna designs proposed therein are based on the electronically steerable parasitic array receptor (ESPAR) antenna [15] which constitutes a low-hardware complexity smart-antenna system implemented by a set of tunable reactive loads connected to a group of parasitic elements coupled to a single central active element.

The present work aims at studying the spectrum sensing performance using smart switched-beam antenna systems. The study considers the single-radio ESPAR antenna structure for the $\mathrm{CR}$ receiver whereas the simple energy detection is the deployed sensing algorithm. The purpose is to detect the existence of a PU signal in the vicinity of the SU receiver as shown in Fig. 1. A statistical analytical channel model is assumed where the multiple signal paths are organized into wave clusters so that the PU signal impinges on the SU receiver through a set of $P$ clusters each of a certain mean angle of arrival (AoA) and angle spread (AS). The reactive loads and the load impedance of the ESPAR receive antenna system are optimized for maximum efficiency and 


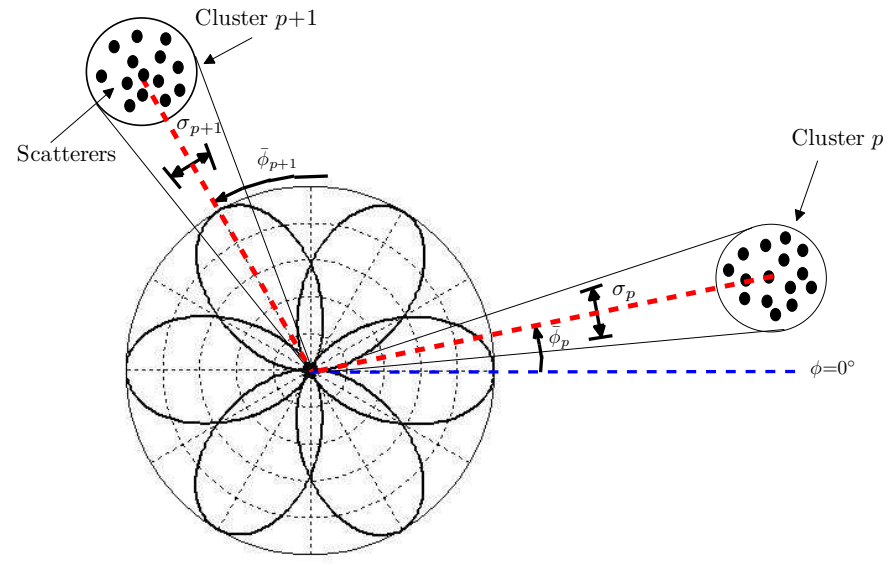

Fig. 1. Spectrum sensing of primary user signal in a clustered propagation scenario using a switched-beam receive antenna system.

directivity in the beampattern look direction. At a given frequency, circular permutations of the optimal reactive loads of the ESPAR antenna rotate the beampattern to different angular positions dividing the whole space around the cognitive receiver into several angular subspaces. At each position, PU signal detection is performed over the directional array diagram. It is shown that beampattern directionality leverages the performance of energy detection by enhancing the receive signal-to-noise ratio (SNR). The detection performance is evaluated under different propagation conditions. The paper also considers the case where, after a full space scan with energy detection being deployed sequentially on every pattern, beam selection combining takes place by choosing the beam with the highest receive signal energy. The performance of the scheme is evaluated in a practical clustering environment showing that the selection combining across the weakly correlated beampatterns gives rise to a diversity action further boosting the probability of detection.

The rest of the paper is organized as follows. Section II gives the signal model and describes the radio environment. Section III presents the ESPAR receive antenna system. In Section IV spectrum sensing over the ESPAR directional beam response is presented and evaluated under various propagation conditions. Section V describes and evaluates the beampattern selection combining method and Section VI concludes the paper.

Notation: Boldface capital letters refer to matrices and boldface small letters refer to vectors of the specified size. $\langle\cdot\rangle_{i j}$ returns the $\{i, j\}$ entry of the enclosed matrix and $\langle\cdot\rangle_{i}$ returns the $i$ th element of the enclosed vector. $\mathbb{C}$ denotes the set of complex numbers of the specified dimensions and $|\cdot|$ gives the amplitude of the enclosed complex number. diag $(\cdot)$ denotes the diagonal matrix whose diagonal entries are the elements of the enclosed vector. The superscripts $\mathrm{T}, *$ and $\mathrm{H}$ denote transpose, conjugate and transpose conjugate, respectively. $\mathcal{R}\{\cdot\}$ denotes the real part of the enclosed complex number and $\mathrm{j}$ is the imaginary unit $\left(\mathrm{j}^{2}=-1\right)$.

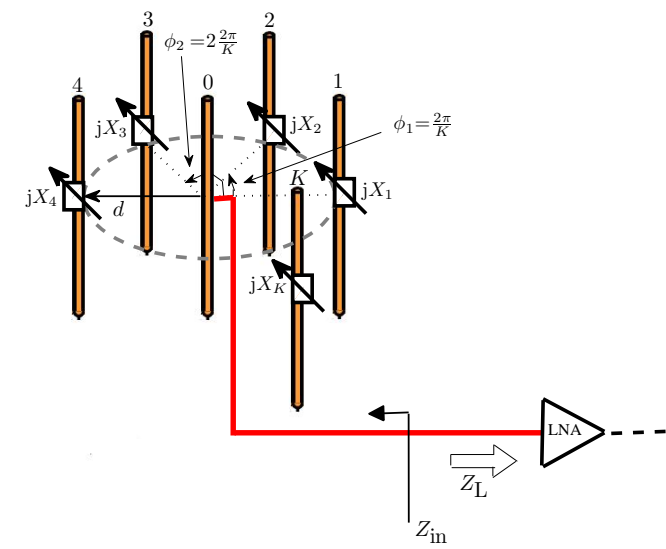

Fig. 2. Configuration of $(K+1)$-element ESPAR receive antenna.

\section{Signal Model}

We focus on two-dimensional propagation in the horizontal (azimuth) plane. The antenna system can switch among $K$ directional beampatterns. The look direction and the free-space response of the $k$ th complex beampattern is $\phi_{k}=(k-1) \frac{2 \pi}{K}$ and $\mathcal{B}_{k}(\phi), k=1, \ldots, K$, respectively, where $\phi$ is the azimuth angle expressing the AoA of the PU signal. The probability density function (pdf) of the angular distribution of impinging PU waves (rays) within a cluster, i.e., the power angle spectrum (PAS), is denoted by $\mathcal{A}(\phi)$. The PAS of a wave cluster is parameterized by a mean AoA $\bar{\phi}$ and AS $\sigma$ (e.g., in case of a Laplacian PAS $\mathcal{A}(\phi)=\frac{c}{\sigma} e^{-|\phi-\bar{\phi}| / \sigma}$, where $c=\frac{\sigma}{\int_{0}^{2 \pi} e^{-|\phi-\bar{\phi}|} d \phi}$ is a normalization factor). Analyzing the directivity gain in the presence of angular distribution of the impinging waves invokes calculating the distributed directivity gain (DDG) (average beamforming gain) at the $k$ th beampattern as [16]

$$
G_{k}(\phi)=\eta \underbrace{\frac{\int_{0}^{2 \pi} \mathcal{B}_{k}(\varphi) \mathcal{B}_{k}^{*}(\varphi) \mathcal{A}(\varphi-\phi) d \varphi}{\frac{1}{2 \pi} \int_{0}^{2 \pi} \mathcal{B}_{k}(\varphi) \mathcal{B}_{k}^{*}(\varphi) d \varphi}}_{D_{k}(\phi)},
$$

where $D_{k}(\phi)$ is the directivity and $\eta \in[0,1]$ is the efficiency of the antenna system.

The received passband signal waveform at the $k$ th beampattern in the presence of a primary user signal is

$$
y_{k}(t)=\mathcal{R}\left\{\left[\tilde{y}_{k}(t)+n_{k}(t)\right] e^{\mathrm{j} 2 \pi f t}\right\},
$$

where $f$ is the carrier frequency and $\tilde{y}_{k}(t) \in \mathbb{C}$ is the PU signal received at the $k$ th beampattern including the effects of fading where the multipath reflections and/or the scatterers are grouped into clusters and modeled as a single path with an associated AS. Let $N_{0}$ denote the one-sided flat noise power spectral density (PSD) and $W$ the signal bandwidth. $n_{k}(t) \in$ $\mathbb{C}$ is the 0 -mean Gaussian noise process with variance $\sigma_{\mathrm{n}}^{2}=$ $N_{0} W$ and is assumed to be white both temporally and spatially (i.e., across the different antenna patterns). The noise and the signal are assumed uncorrelated. 
In (2), $\tilde{y}_{k}(t)$ is the superposition of rays arriving from $P$ power-balanced wave clusters. Specifically, it is assumed that the unit energy PU signal $s(t)$ impinges on $P$ clusters, where the $p$ th cluster has a Laplacian PAS $\mathcal{A}_{p}(\phi)$, mean AoA $\bar{\phi}_{p}$ and AS $\sigma_{p}, p \in\{1, \ldots, P\}$ (Fig. 1). Thus, the PU signal received at the $k$ th beampattern can be written as

$$
\tilde{y}_{k}(t)=\sqrt{\frac{P_{\mathrm{T}}}{P}} \sum_{p=1}^{P} \sqrt{G_{k}\left(\bar{\phi}_{p}\right)} h_{k, p} s(t),
$$

where $P_{\mathrm{T}}$ is the PU transmit power and $h_{k, p} \in \mathbb{C}$ is the slowfading channel coefficient for the $p$ th PU signal received at the $k$ th beampattern. The receive SNR and the total average DDG at the $k$ th beampattern are respectively given by

$$
\begin{gathered}
\gamma_{k}=\frac{P_{\mathrm{T}}}{P}\left[\sum_{p=1}^{P} G_{k}\left(\bar{\phi}_{p}\right)\left|h_{k, p}\right|^{2}\right] / \sigma_{\mathrm{n}}^{2}, \\
G_{k}=\sum_{p=1}^{P} G_{k}\left(\bar{\phi}_{p}\right) / P .
\end{gathered}
$$

For $\sigma_{p}=0^{\circ}$ and for deterministic $h_{k, p}$ the signal model in (3) accounts for the simple case of additive white Gaussian noise (AWGN) channel or, equivalently, free-space propagation.

\section{ESPAR RECEIVE ANTENNA DESCRIPTION}

We consider directional sensing using a single-radio $(K+1)$ element ESPAR receive antenna (Fig. 2). The ESPAR is a smart antenna system that presents a significant advantage over its directional antenna counterparts: it is able to control its beampatterns as any smart antenna, while being implemented using a single active element (element 0) surrounded by $K$ passive (parasitic) elements (elements $\{1, \ldots, K\}$ ). The $k$ th parasitic element is placed at a relative local angle of $\phi_{k}=(k-1) \frac{2 \pi}{K}, k \in\{1, \ldots, K\}$. The parasitic elements are terminated with varactors controlling the imaginary part of their input impedances. The free-space beampattern is

$$
\mathcal{B}_{k}(\phi)=\mathbf{w}_{\mathrm{eq}, k}^{\mathrm{T}} \boldsymbol{\alpha}(\phi) .
$$

In (6), $\boldsymbol{\alpha}(\phi) \in \mathbb{C}^{(K+1) \times 1}$ is the array steering vector resulting from the array geometry as

$$
\boldsymbol{\alpha}(\phi)=\left[\begin{array}{llll}
1 & e^{-\mathrm{j} \kappa d \cos (\phi)} & \ldots & e^{-\mathrm{j} \kappa d \cos (\phi-(K-1)(2 \pi / K))}
\end{array}\right]^{\mathrm{T}},
$$

where $d$ is the radius of the ESPAR, equal to the spacing between the central active element and the parasitic elements, and $\kappa=2 \pi / \lambda$ is the wavenumber. $\mathbf{w}_{\mathrm{eq}, k} \in \mathbb{C}^{(K+1) \times 1}$ is the equivalent weight vector at which the $k$ th beampattern is created and is given by

$$
\mathbf{w}_{\mathrm{eq}, k}=\left[\mathbf{Z}+\mathbf{X}_{k}\right]^{-1} \mathbf{u},
$$

where $\mathbf{Z} \in \mathbb{C}^{(K+1) \times(K+1)}$ is the mutual impedance matrix whose entry $\langle\mathbf{Z}\rangle_{k k}$ is the self-impedance of the $k$ th antenna element and $\langle\mathbf{Z}\rangle_{k \ell}, k \neq \ell$ is the mutual impedance between the $k$ th and the $\ell$ th antenna element, where $\langle\mathbf{Z}\rangle_{k \ell}=$ $\langle\mathbf{Z}\rangle_{\ell k}, k, \ell \in\{0,1, \ldots, K\}$ by reciprocity. The vector $\mathbf{u}$ is given by $\mathbf{u}=[1 \underbrace{0 \ldots 0}_{K}]^{\mathrm{T}}$.
Let the loading vector $\mathbf{x}=\left[\begin{array}{lll}X_{1} & X_{2} \ldots X_{K}\end{array}\right]^{\mathrm{T}} \Omega$ denote the set of $K$ loads to be mapped to the $K$ parasitic elements, such that the circular rotation of the loading vector rotates the beampattern to one of the $K$ angular positions (based on the image theory). Then, the matrix $\mathbf{X}_{k} \in \mathbb{C}^{(K+1) \times(K+1)}$ can be defined as

$$
\mathbf{X}_{k}:=\operatorname{diag}\left(\left[Z_{\mathrm{L}} \mathrm{j} \hat{\mathbf{x}}_{k}\right]\right)
$$

where $\hat{\mathbf{x}}_{k} \in\{1, \ldots, K\}$ is a circular permutation of $\mathbf{x}$ at which the $k$ th beampattern is created so that the $\ell$ th element of $\hat{\mathbf{x}}_{k}$ is given by $\left\langle\hat{\mathbf{x}}_{k}\right\rangle_{\ell}=X_{1+[(k+\ell-2) \bmod K]}$. In (9), $Z_{\mathrm{L}}$ is the is the load impedance of the receiver, i.e., the output impedance of the low-noise amplifier (LNA).

Assuming negligible ohmic losses, any losses in the ESPAR system may result from the mismatch between the source impedance and the input impedance seen by the central active antenna. The input impedance $Z_{\text {in }}$ of the active element and the corresponding reflection efficiency because of impedance mismatch can be respectively written as

$$
\begin{aligned}
& Z_{\text {in }}=\langle\mathbf{Z}\rangle_{00}+\frac{1}{\left\langle\mathbf{i}_{k}\right\rangle_{0}} \sum_{\ell=1}^{K}\langle\mathbf{Z}\rangle_{0 \ell}\left\langle\mathbf{i}_{k}\right\rangle_{\ell}, \\
& \eta=1-\left|\left(Z_{\text {in }}+Z_{\mathrm{L}}\right)^{-1}\left(Z_{\text {in }}-Z_{\mathrm{L}}^{*}\right)\right|^{2},
\end{aligned}
$$

respectively, where $\mathbf{i}_{k}=\mathbf{w}_{\mathrm{eq}, k} v_{\mathrm{T}} \in \mathbb{C}^{(K+1) \times 1}$ is the vector of induced currents on the antenna elements. $Z_{\text {in }}$ remains constant $\forall k$ and thus $\eta$ is maintained $\forall \mathcal{B}_{k}(\phi), k \in\{1, \ldots, K\}$, due to topology symmetry.

\section{Spectrum Sensing OVER DiRECTIONAL BEAM RESPONSE}

In order to analyze the sensing performance using directional CR receiver, the low-complexity energy detection is used for spectrum sensing. The goal of spectrum sensing is to decide between the two hypotheses over the $k$ th beampattern

$$
y_{k}(t)=\left\{\begin{array}{cc}
\mathcal{R}\left\{n(t) e^{\mathrm{j} 2 \pi f t}\right\}, & \mathcal{H}_{0} \\
\mathcal{R}\left\{\left[\tilde{y}_{k}(t)+n_{k}(t)\right] e^{\mathrm{j} 2 \pi f t}\right\}, & \mathcal{H}_{1} .
\end{array}\right.
$$

The test or decision statistic is equal to the receive energy over the observation time interval $(0, T)$ over the $k$ th beampattern and can be approximated as [6]

$$
\mathcal{T}_{k}=\int_{0}^{T} y_{k}^{2}(t) \approx \frac{1}{2 W} \sum_{i=1}^{2 W T} y_{k}^{2}\left(\frac{i}{2 W}\right),
$$

where $y_{k}\left(\frac{i}{2 W}\right)$ is the $i$ th sample of $y_{k}(t)$ and $N=2 W T$ is the number of samples ${ }^{1}$. When the PU signal is not present (under hypothesis $\mathcal{H}_{0}$ ), the decision statistic is the sum of the squares of $2 W T$-mean Gaussian distributed random variables with variance $\sigma_{\mathrm{n}}^{2}$, thus $\mathcal{T}_{k}$ will follow a central chi-square distribution. Based on the cumulative distribution function $(\mathrm{CDF})$ of $\mathcal{T}_{k}$, the probability of false alarm is given by [7]

$$
P_{\mathrm{f}}=\operatorname{Pr}\left(\mathcal{T}_{k}>\delta \mid \mathcal{H}_{0}\right)=\Gamma\left(W T, \delta / 2 \sigma_{\mathrm{n}}^{2}\right) / \Gamma(W T),
$$

\footnotetext{
${ }^{1}$ It is assumed that the that the first sample position starts at $t=0$.
} 
where $\delta$ is the detection threshold, $\Gamma(a)$ is the Gamma function and $\Gamma(a, b)$ is the unregularized upper incomplete Gamma function [17].

Similarly, when the PU signal is present (under hypothesis $\mathcal{H}_{1}$ ) and for AWGN channels, the decision statistic $\mathcal{T}_{k}$ has a chi-square distribution with a non centrality parameter $2 W T \gamma_{k}$ [6]. Based on the statistics of $\mathcal{T}_{k}$, the probability of detection over the $k$ th beampattern is given by

$$
P_{\mathrm{d}, k}=\operatorname{Pr}\left(\mathcal{T}_{k}>\delta \mid \mathcal{H}_{1}\right)=Q_{W T}\left(\sqrt{2 W T \gamma_{k}}, \sqrt{\delta / \sigma_{\mathrm{n}}^{2}}\right),
$$

where $Q_{z}(a, b)$ is the generalized Marcum $Q$-function [17]. Under Rayleigh fading, the receive SNR follows an exponential probability density function (PDF) $f\left(\gamma_{k}\right)=$ $\left(1 / \bar{\gamma}_{k}\right) e^{\left(-\gamma_{k} / \bar{\gamma}_{k}\right)}$, where $\bar{\gamma}_{k}$ is the average receive SNR. Thus, the average $P_{\mathrm{d}, k}$ is given by [5]

$$
\begin{aligned}
& \bar{P}_{\mathrm{d}, k}=1- \Gamma^{\prime} \\
&\left(W T, \frac{\delta}{2}\right)+\left(\frac{\bar{\gamma}_{k}^{2}+1}{\bar{\gamma}_{k}^{2}}\right)^{W T} . \\
& e^{\frac{\delta}{2\left(\bar{\gamma}_{k}^{2}+1\right)}} \Gamma^{\prime}\left(W T, \frac{\delta \bar{\gamma}_{k}^{2}}{2\left(\bar{\gamma}_{k}^{2}+1\right)}\right),
\end{aligned}
$$

where $\Gamma^{\prime}(a, b)=\Gamma(a, b) / \Gamma(a)$ is the regularized upper incomplete Gamma function.

Although the probability of false alarm in (14) is not related to the receive SNR (and the receiving beampattern) as there is no signal, the probability of detection is a function of $\gamma_{k}$ given in (4). Thus, the performance of sensing in terms of probability of detection can be improved by the DDG of the directional beampattern enhancing the receive SNR. To illustrate, we evaluate the sensing performance over a beampattern of specific ESPAR configurations with respect to a standard isotropic receiver $(G(\phi)=0 \mathrm{dBi})$. In these examples, we take into account sensing over the 1 st $(k=1)$ beampattern (the analysis for other beampatterns is similar). We consider ESPAR set-ups of $K+1=3$ and $K+1=7$ identical half-wavelength thin electrical dipoles with $d=\lambda / 4$, where $\lambda$ is the carrier free-space wavelength. The mutual impedance matrix $\mathbf{Z}$ of the 3-port receive antenna system has $\langle\mathbf{Z}\rangle_{00}=73.07+\mathrm{j} 42.50,\langle\mathbf{Z}\rangle_{01}=40.75-\mathrm{j} 28.32$, and $\langle\mathbf{Z}\rangle_{12}=-12.52-\mathrm{j} 29.90$, calculated using Gauss-Legendre numerical integration [18]. Similarly, the 7-element ESPAR has $\langle\mathbf{Z}\rangle_{00}=73.07+\mathrm{j} 42.50,\langle\mathbf{Z}\rangle_{01}=40.75-\mathrm{j} 28.32$, $\langle\mathbf{Z}\rangle_{13}=-0.66-\mathrm{j} 35.93$ and $\langle\mathbf{Z}\rangle_{14}=-12.52-\mathrm{j} 29.90^{2}$. The ESPAR systems are optimized with respect to $Z_{\mathrm{L}}$ and $\mathbf{x}$ for maximum DDG in the look direction of the array diagram, i.e., for maximum $G_{1}\left(0^{\circ}\right)$, via a constrained nonlinear optimization multivariate MATLAB routine yielding the optimized antenna parameters in Table 1 . The 1 st array pattern $\mathcal{B}_{1}(\phi)$ of the optimized 7-element ESPAR is shown in Fig. 4(a). It should be noted that the remaining optimal beampatterns $\mathcal{B}_{k}(\phi)$, $k=\{2, \ldots, K\}$ (i.e., with maximum DDG in their look direction) are simply obtained by appropriately rotating the reactive loads, according to Section III.

It is considered that a single PU signal $(P=1)$ is arriving over an AWGN channel and that the direction of arrival is aligned with the look direction of the beam $\left(\bar{\phi}_{1}=0^{\circ}\right)$. The

\footnotetext{
${ }^{2}$ The other entries of the $\mathbf{Z}$ matrices can be easily obtained from the antenna topology symmetry.
}

\begin{tabular}{|c|c|c|c|}
\hline$(K+1)$ & $\begin{array}{c}\text { Optimal load } \\
\text { impedance } Z_{\mathrm{L}}(\Omega)\end{array}$ & $\begin{array}{l}\text { Optimal reactive loads } \\
\mathbf{x}(\Omega)\end{array}$ & $\begin{array}{c}G_{1}\left(0^{\circ}\right) \\
(\mathrm{dBi})\end{array}$ \\
\hline 3 & $90.2-\mathrm{j} 97.6$ & {$\left[\begin{array}{ll}92.4 & 6.7\end{array}\right]^{\mathrm{T}}$} & 4.0 \\
\hline 7 & $52.3-\mathrm{j} 82.3$ & $\begin{array}{ccc}{[-33.4} & -160.7 & 80.7 \\
-160.1 & -33.2 & -71.9]^{\mathrm{T}} \\
\end{array}$ & 7.0 \\
\hline
\end{tabular}

TABLE I

OPTIMIZED ESPAR PARAMETERS

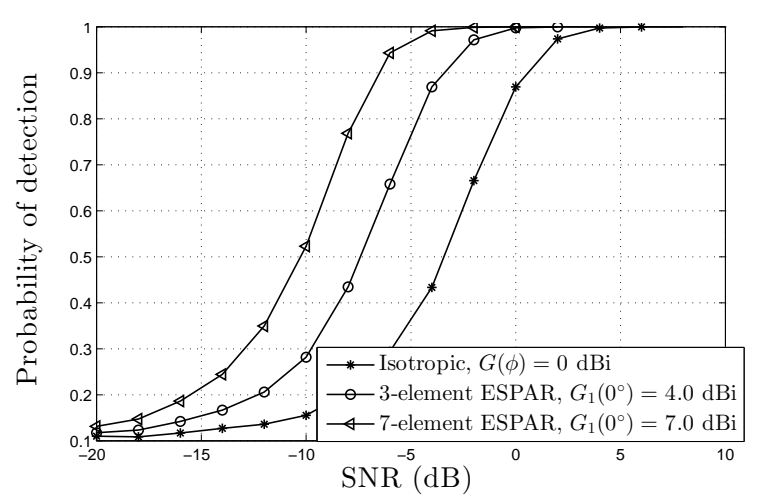

Fig. 3. Probability of detection versus SNR for spectrum sensing over the directional ESPAR beam response $\left(P_{\mathrm{f}}=0.1, W T=10\right)$.

probability of detection averaged over 10,000 random channel drops (Monte-Carlo simulations) versus the input SNR for $W T=10$ and $P_{\mathrm{f}}=0.1$ is shown in Fig. 3. It can be observed that sensing with the directional optimized ESPAR systems significantly improves the detection performance, resulting in SNR gain of up to 4 and $8 \mathrm{~dB}$ with the 3 -element ESPAR and the 7-element ESPAR, respectively.

In practice, the ideal assumption that the PU mean AoA is aligned with the beam look direction may not be fulfilled. From (4) and (15) or (16), it can be seen that the probability of detection depends on the mean AoA of the received PU signal(s). Moreover, it is realistic to account for more severe channel impairments to the signal reception than simple AWGN, such as the multipath Rayleigh fading. Thus, in this part a single PU signal is assumed arriving from various possible mean AoAs (Fig. 4(a)) on the aforementioned optimized 7-element ESPAR receive antenna under Rayleigh fading and for $\sigma_{p}=0^{\circ}$. The calculated DDG $G\left(\bar{\phi}_{1}\right)$ decreases from $7.0 \mathrm{dBi}$ to $-0.2 \mathrm{dBi}$ for $\bar{\phi}_{1}$ varying from $0^{\circ}$ to $50^{\circ}$. The receiver operating characteristic (ROC) $\left(P_{\mathrm{d}, 1}\right.$ versus $P_{\mathrm{f}}$ ), or equivalently, the complementary ROC (probability of miss detection $P_{\mathrm{m}, 1}=1-P_{\mathrm{d}, 1}$ versus $P_{\mathrm{f}}$ ) for the different situations is shown in Fig. 4(b). The input SNR is $-7 \mathrm{~dB}$. It can be observed, that as the mean AoA deviates from the value $\bar{\phi}_{1}=0^{\circ}$ at which the beampattern has bean optimized, the attained DDG decreases, with a direct impact on the sensing performance.

Furthermore, a non-zero AS can also affect the sensing performance. Here it is assumed that the mean AoA of the PU signal is kept at $\bar{\phi}=0^{\circ}$ whereas the $\mathrm{AS} \sigma_{1}$ varies from $0^{\circ}$ to $50^{\circ}$. As the AS increases (Fig. 5(a)), the beampatern diffuses and the lobes start to smear, whereas the DDG at the look direction decreases. At high values of AS (e.g., for $\sigma_{1}=50^{\circ}$ with $G_{1}\left(0^{\circ}\right)=4.0 \mathrm{dBi}$ ) the array diagram tends to flatten and tends to approach an omnidirectional diagram, indicating that 


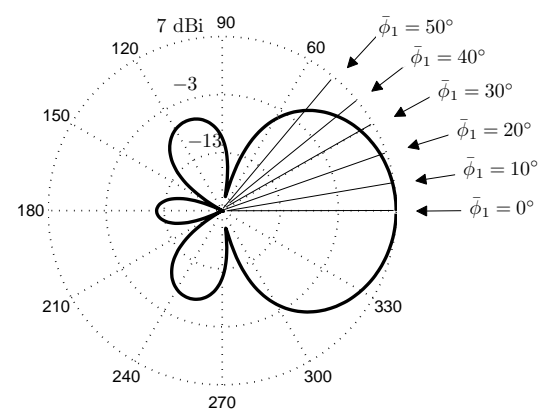

(a)

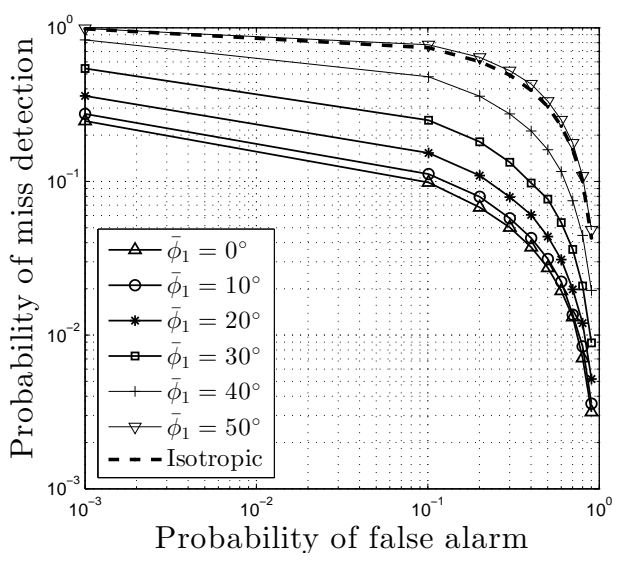

(b)

Fig. 4. Performance of directional sensing for different values of mean AoA $\bar{\phi}$. (a): Array diagram $\mathcal{B}_{1}(\phi)$ of the optimized 7-element ESPAR and an impinging PU signal with various mean AoA. (b): Complementary ROC curve under Rayleigh fading $(-7 \mathrm{~dB}$ SNR, $W T=10)$.

beamforming gains under rich scattering environments are not so significant. Fig. 5(b) shows that the sensing performance is affected in a way similar to the case of mean AoA misalignment with the beam look direction.

TABLE II

DDG OF THE OPTIMIZED 7-ELEMENT ESPAR ARRAY

$\begin{array}{lccc} & \bar{\phi}_{1}=5^{\circ} & \bar{\phi}_{2}=130^{\circ} \\ G_{1}\left(\bar{\phi}_{p}\right)(\mathrm{dBi}) & 7.0 & -8.6 \\ G_{2}\left(\bar{\phi}_{p}\right)(\mathrm{dBi}) & -2.5 & -11.4 \\ G_{3}\left(\bar{\phi}_{p}\right)(\mathrm{dBi}) & -7.0 & 6.7 \\ G_{4}\left(\bar{\phi}_{p}\right)(\mathrm{dBi}) & -11.8 & -0.5 \\ G_{5}\left(\bar{\phi}_{p}\right)(\mathrm{dBi}) & -7.4 & -6.8 \\ G_{6}\left(\bar{\phi}_{p}\right)(\mathrm{dBi}) & -7.3 & -12.6\end{array}$

\section{Spectrum Sensing via Beam Scanning with BEAM SELECTION COMBINING}

In this section we assume that spectrum sensing is run sequentially on each one of the $K$ ESPAR optimized beampatterns using energy detection as described in the previous

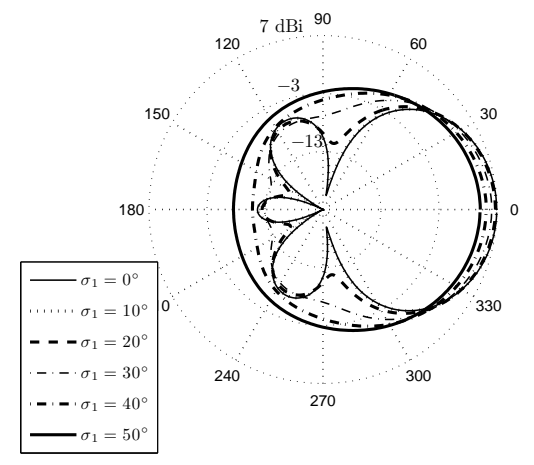

(a)

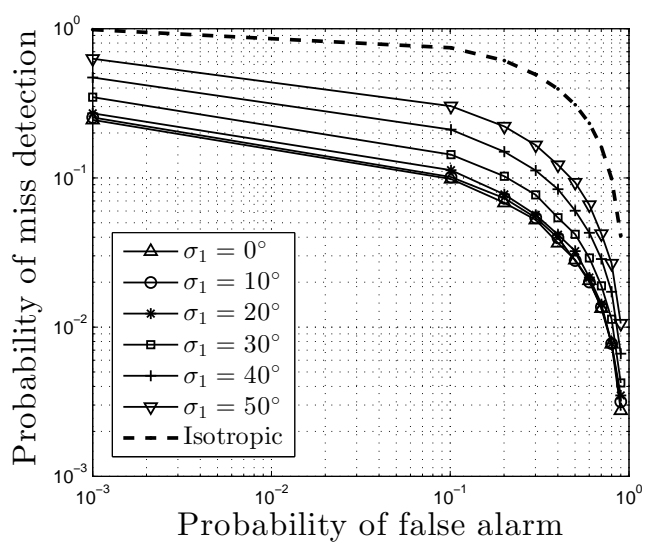

(b)

Fig. 5. Performance of directional sensing for different values of AS $\sigma$. (a): Array diagram $\mathcal{B}_{1}(\phi)$ of the optimized 7 -element ESPAR for various values of AS of the impinging PU signal. (b): Complementary ROC curve under Rayleigh fading $(-7 \mathrm{~dB}$ SNR, $W T=10)$.

section under Rayleigh fading. It is assumed that the channel fading coefficient remains unchanged during each full angular space scan whereas it changes to another independent realization in a succeeding scan. This assumption is valid for slow fading channels while assuming that beam scanning and detection over each array diagram happens fast enough. After a full space scan, the receiver performs beam selection based on the gathered $K$ test statistics $\mathcal{T}_{k}, k \in\{1, \ldots, K\}$. Specifically, the maximum $\mathcal{T}_{k}, k \in\{1, \ldots, K\}$ is chosen and compared with the detection threshold $\delta$ to perform the sensing test and the combined probability of detection $\bar{P}_{\mathrm{d}, \mathrm{SC}}$ is thus given by

$$
\bar{P}_{\mathrm{d}, \mathrm{SC}}=\operatorname{Pr}\left(\max _{k \in\{1, \ldots, K\}} \mathcal{T}_{k}>\delta \mid \mathcal{H}_{1}\right) .
$$

Optimizing the beampatterns for the maximum DDG at the look direction has the additional advantage of resulting in a sufficiently weak correlation across the $K$ array diagrams. In the general case, the signal model accounting for beampattern cross-correlation (under hypothesis $\mathcal{H}_{1}$ ) can be written as

$$
\mathbf{y}(t)=\sqrt{P_{\mathrm{T}}}\left(\mathbf{R}_{\mathrm{R}}\right)^{1 / 2} \mathbf{h} s(t)+\mathbf{n}(t),
$$


where $\mathbf{y}(t) \in \mathbb{C}^{K \times 1}$ is the vector of the $K$ collected received signals upon a beam scan. $\mathbf{h} \in \mathbb{C}^{K \times 1}$ is the vector of slow fading channel coefficients with $\langle\mathbf{h}\rangle_{k}=\sum_{p=1}^{P} h_{k, p}$ and $\mathbf{n}(t) \in \mathbb{C}^{K \times 1}$ is the temporally and spatially white noise vector with $\langle\mathbf{n}(t)\rangle_{k}=n_{k}(t) . \mathbf{R}_{\mathbf{R}} \in \mathbb{C}^{K \times K}$ is the receive covariance matrix that incorporates both the power imbalance and the cross-correlation of the beampatterns

$$
\mathbf{R}_{\mathrm{R}}=\mathbf{G}^{1 / 2} \tilde{\mathbf{R}} \mathbf{G}^{1 / 2},
$$

where $\mathbf{G}=\operatorname{diag}\left[G_{1} \quad \ldots \quad G_{K}\right]$ and the normalized correlation matrix $\tilde{\mathbf{R}} \in \mathbb{C}^{K \times K}$ has entries

$$
\begin{aligned}
\langle\tilde{\mathbf{R}}\rangle_{k j} & =\frac{\left\langle\mathbf{R}_{\mathrm{R}}\right\rangle_{k j}}{\sqrt{\left\langle\mathbf{R}_{\mathrm{R}}\right\rangle_{k k}\left\langle\mathbf{R}_{\mathrm{R}}\right\rangle_{j j}}} \\
& =\frac{\int_{0}^{2 \pi} \mathcal{B}_{k}(\phi) \mathcal{B}_{j}^{*}(\phi) \mathcal{A}_{\Sigma}(\phi) d \phi}{\sqrt{\int_{0}^{2 \pi} \mathcal{B}_{k}(\phi) \mathcal{B}_{k}^{*}(\phi) \mathcal{A}_{\Sigma}(\phi) d \phi \int_{0}^{2 \pi} \mathcal{B}_{j}(\phi) \mathcal{B}_{j}^{*}(\phi) \mathcal{A}_{\Sigma}(\phi) d \phi}},
\end{aligned}
$$

where $\mathcal{A}_{\Sigma}(\phi)=\sum_{p=1}^{P} \mathcal{A}_{p}(\phi) / P$ is the total PAS.

To illustrate, we consider the 7-element ESPAR antenna system of Section IV optimized for maximum DDG at the look direction (Table I) under Rayleigh fading. It is assumed that $P=2$ PU signals arrive on the array through two different clusters with Laplacian PAS $\mathcal{A}_{1}(\phi)$ and $\mathcal{A}_{2}(\phi)$ with $\bar{\phi}_{1}=5^{\circ}$ and $\bar{\phi}_{2}=130^{\circ}$, respectively, whereas $\sigma_{1}=\sigma_{2}=10^{\circ}$. These parameters describe a typical indoor clustered propagation scenario and are similar to those used in the IEEE 802.11 wireless local area network Task Group N [19]. The attained DDGs at every beampattern at the mean AoA of the PU clusters are summarized in Table II. The probability of detection versus the input SNR of spectrum sensing with beam selection via the 7-element ESPAR for $P_{\mathrm{f}}=0.1$ is shown in Fig. 6 . The resulting maximum absolute cross-correlation coefficient is $\max \left|\langle\mathbf{R}\rangle_{k j}\right|=0.7$. The figure also shows sensing only over the 1st beampattern $\mathcal{B}_{1}(\phi)$ and over a standard isotropic antenna. It can be observed that sensing over $\mathcal{B}_{1}(\phi)$ improves the probability of detection due to receive SNR gain as discussed in Section IV. However, beam scanning with beam selection combining, along with receive SNR improvement due to the beampattern directionality, can enable a diversity action that further boosts the probability of detection. Moreover, the performance approaches the ideal beam selection with uncorrelated beampatterns, indicating that indeed the designed beampatterns are approximately orthogonal.

\section{CONCLUSION}

The paper has described directional spectrum sensing using the single-radio switched-beam ESPAR antenna where PU signal detection is performed on each antenna pattern. The antenna's loading conditions have been optimized for maximum average beamforming gain in the beampattern look direction. The simulation results showed that the average beamforming gain of the beam response can improve the probability of detection of the energy detector by enhancing the SNR at the receiver side. Moreover, by selectively combining the sensing results from the $K$ weakly correlated beampatterns diversity gains can be added to the cognitive radio system performance.

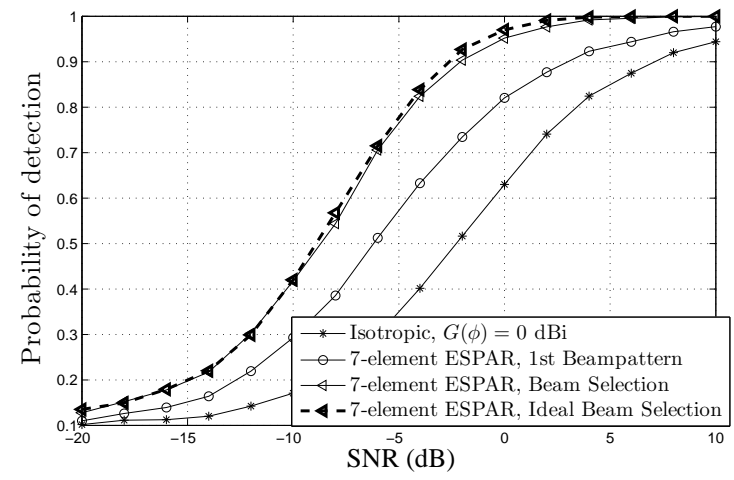

Fig. 6. Probability of detection versus SNR for beam scanning with beam selection combining under Rayleigh fading $\left(P_{\mathrm{f}}=0.1, W T=10\right)$.

\section{REFERENCES}

[1] S. Haykin, "Cognitive radio: Brain-empowered wireless communications," IEEE J. Sel. Areas in Commun., vol. 23, no. 2, pp. 201-220, 2005.

[2] T. Yucek and H. Arslan, "A survey of spectrum sensing algorithms for cognitive radio applications," IEEE Commun. Surveys and Tutorials, vol. 11, no. 1, pp. 116-130, 2009.

[3] S. M. Kay, Fundamentals of Statistical Signal Processing: Detection Theory, vol. 2. Prentice Hall, 1998.

[4] W. A. Gardner, "Exploitation of spectral redundancy in cyclostationary signals," IEEE Signal Processing Mag., vol. 8, pp. 14-36, 1991.

[5] V. I. Kostylev, "Energy detection of a signal with random amplitude," IEEE Int. Conf. on Commun. (ICC '02), pp. 1606-1610, May 2002.

[6] F. F. Digham, M.-S. Alouini, and M. K. Simon, "On the energy detection of unknown signals over fading channels," IEEE Int. Conf. on Commun. (ICC '03), vol. 5, pp. 3573-3579, May 2003.

[7] F. F. Digham, M.-S. Alouini, and M. K. Simon, "On the energy detection of unknown signals over fading channels," IEEE Trans. Commun. vol. 55, no. 1, pp. 21-24, 2007.

[8] Y. Zeng, Y.-C. Liang, E. C. Y. Peh, and A. T. Hoang, "Cooperative covariance and eigenvalue based detections for robust sensing," IEEE Global Commun. Conf. (GLOBECOM '09), pp. 1-6, Nov. 2009.

[9] R. Zhang, T. Lim, Y.-C. Liang, and Y. Zeng, "Multi-antenna based spectrum sensing for cognitive radios: A GLRT approach," IEEE Trans. Commun., vol. 58, no. 1, pp. 84-88., 2010.

[10] H. Sarvanko, M. Hoyhtya, M. Matinmikko, and A. Mammela, "Exploiting spatial dimension in cognitive radios and networks ,"6th Int'l ICST Conf. Cognitive Radio Oriented Wireless Networks and Commun. (CROWNCOM'11), pp. 360-364, 2011.

[11] S. Huang; Z. Ding, X. Liu, "Non-intrusive cognitive radio networks based on smart antenna technology," IEEE Global Telecommun. Conf. (GLOBECOM '07), pp. 4862-4867, 2007.

[12] K.-L. Du and W. H. Mow, "Affordable cyclostationarity-based spectrum sensing for cognitive radio with smart antennas," IEEE Trans. Vehicular Technology, vol. 59, no. 4, pp. 1877-1886, 2010.

[13] E. P. Tsakalaki, O. N. Alrabadi, C. B. Papadias, R. Prasad, "Spatial spectrum sensing for wireless handheld terminals: design challenges and novel solutions based on tunable parasitic antennas," IEEE Wireless Commun. Mag., special issue on Dynamic Spectrum Management in Wireless Networks, vol. 17, no. 4, pp. 33-40, 2010.

[14] E. P. Tsakalaki, O. N. Alrabadi, C. B. Papadias, R. Prasad, "Spatial spectrum sensing for cognitive radios via miniaturized parasitic antenna Systems," 5th Int'l ICST Conf. Cognitive Radio Oriented Wireless Networks and Commun. (CROWNCOM '10), pp. 1-5, June 2010.

[15] C. Sun, A. Hirata, T. Ohira, and N. C. Karmakar, "Fast Beamforming of Electronically Steerable Parasitic Array Radiator Antennas: Theory and Experiment," IEEE Trans. Antennas and Propag., vol. 52, no.7, 2004.

[16] R. Vaughan and J. B. Andersen, Channels, Propagation and Antennas for Mobile Communications, IEE Electromagnetic Waves Series, Institution of Electrical Engineers, 2003.

[17] I. S. Gradshteyn and I. M. Ryzhik, Table of Integrals, Series, and Products, 6th ed. San Diego, CA: Academic, 2000.

[18] S. J. Orphanides, Electrom. waves and antennas, Rutgers Univ., 2008.

[19] V. Erceg et al., "TGn channel models," IEEE 802.11 standard contribution 802.11-03/940r4, May 2004. 